\title{
Capítulo 14. Utilidad del mapeo tridimensional en la ablación de taquicardia ventricular en pacientes con miocardiopatía hipertrófica, taquicardia ventricular catecolaminérgica y síndrome de Brugada
}

\author{
Chapter 14. Usefulness of three-dimensional mapping \\ in ablation of ventricular tachycardia in patients \\ with hypertrophic cardiomyopathy, catecholaminergic \\ ventricular tachycardia and Brugada syndrome
}

\author{
Alejandro Jiménez ${ }^{\mathrm{a}}$, Alejandro Olayaa ${ }^{\mathrm{b}, \mathrm{c}, *}$, Natalia Morales $^{\mathrm{d}}$ y Jorge Sánchez \\ a Cardiología/Electrofisiología, Wellington Regional Hospital, Victoria University School Of Medicine, Wellington, Nueva \\ Zelanda \\ b Programa de Especialización en Cardiología, Fundación Universitaria de Ciencias de la Salud, Servicio de Electrofisiología, \\ Hospital Cardiovascular del Niño de Cundinamarca, Bogotá, Colombia \\ ' Electrofisiología, Fundación Santa Fe, Bogotá, Colombia \\ d Fundación Universitaria de Ciencias de la Salud, Bogotá, Colombia
}

Recibido el 17 de noviembre de 2015; aceptado el 18 de enero de 2016

\section{Miocardiopatía hipertrófica}

La miocardiopatía hipertrófica es una enfermedad cardiaca monogénica y es causa importante de muerte súbita de origen arrítmico, falla cardiaca y fibrilación auricular. Desde el punto de vista epidemiológico se considera una enfermedad genética común, pero se cree que aún sigue siendo una subdiagnosticada en gran parte del mundo. Se estima una prevalencia de al menos 1 en cada 500 personas $(0,2 \%)$. El diagnós- tico clínico se basa en el hallazgo de hipertrofia ventricular izquierda sin dilatación de las cavidades ventriculares y sin la presencia de enfermedades sistémicas o cardiacas asociadas que puedan producir hipertrofia ventricular (hipertensión sistémica, estenosis aórtica, entre otras) ${ }^{1}$.

Es causada por mutaciones en más de once genes que codifican los componentes proteicos de los miofilamentos de la sarcómera; $70 \%$ de las mutaciones conocidas se encuentran en dos genes, B-miosina (MYH7) y proteína $\mathrm{C}$ de la miosina

\footnotetext{
*Autor para correspondencia.

Correo electrónico: aolaya2000@hotmail.com (A. Olaya).
} 
(MYBPC3); los demás casos corresponden a alteraciones en la troponina T (TNNT2) y otros genes. La enfermedad se transmite de manera autonómica dominante, aunque se han observado casos a consecuencia de mutaciones de novo $0^{2-5}$.

Es, así mismo, la causa más frecuente de muerte súbita cardiaca en personas jóvenes ( $<30$ años) y puede ser la manifestación clínica inicial de la enfermedad. Aunque los episodios de arritmias ventriculares y muerte súbita tradicionalmente se asocian con ejercicio físico extremo (siendo la primera causa de muerte súbita en deportistas de alto rendimiento), la mayoría de los casos de muerte súbita cardíaca se presentan con mínimo esfuerzo físico o aun en reposo $0^{6-8}$. Estudios en los que se analizaron electrogramas de pacientes portadores de cardiodesfibrilador implantado (CDI) demuestran que el mecanismo arritmogénico de la muerte súbita cardíaca en la miocardiopatía hipertrófica es la taquicardia ventricular y/o fibrilación ventricular primaria ${ }^{6}$. Se cree que la desorganización de la arquitectura de los miofilamentos del músculo cardiaco anormal, junto con áreas de fibrosis miocárdica, es responsable del sustrato arrítmico. El diagnóstico clínico se basa en la sospecha clínica, un electrocardiograma con criterios de hipertrofia ventricular izquierda y el aumento del grosor de las paredes del ventrículo izquierdo mediante ecocardiografía bidimensional y/o resonancia nuclear magnética (RNM) ${ }^{9-11}$.

Respecto a la incidencia de arritmias ventriculares en miocardiopatía hipertrófica, la presentación más común suele ser la muerte súbita cardiaca asociada a fibrilación ventricular, pero en algunos pacientes se puede presentar taquicardia ventricular monomórfica sostenida, que lleva a terapias apropiadas del CDI y recurrencias a pesar del manejo antiarrítmico.

La presencia de taquicardia ventricular monomórfica se explica en aquellos pacientes con fibrosis miocárdica marcada. La mayoría de la evidencia sobre la utilidad de la ablación de taquicardia ventricular monomórfica en miocardiopatía hipertrófica se limitaba a casos esporádicos reportados. Más recientemente se han publicado dos estudios multicéntricos ${ }^{12,13}$ en los que se demuestra la utilidad de las técnicas de mapeo tridimensional y del abordaje endoepicárdico para caracterizar el sustrato arrítmico y facilitar la ablación por radiofrecuencia en taquicardia ventricular monomórfica en pacientes con miocardiopatía hipertrófica, donde el 70 al 80\% presentan taquicardia ventricular con circuitos epicárdicos. En el seguimiento a largo plazo (18 a 36 meses), la recurrencia de taquicardia ventricular fue del $30 \%$, similar a los hallazgos reportados para la ablación de taquicardia ventricular en pacientes con cardiopatía isquémica y otras cardiopatías no isquémicas ${ }^{14}$. Estos estudios demuestran que los circuitos de taquicardia ventricular en pacientes con miocardiopatía hipertrófica involucran tanto al epicardio como al endocardio. Debido a la incidencia alta de cicatriz epicárdica y al grosor de la pared ventricular izquierda en pacientes con miocardiopatía hipertrófica, la ablación endoepicárdica ofrece la mejor alternativa terapéutica para el manejo de la taquicardia ventricular recurrente a pesar del tratamiento antiarrítmico en pacientes con miocardiopatía hipertrófica, portadores de CDI. En la evaluación electrofisiológica de estos circuitos, es fundamental el uso de sistemas de mapeo con reconstrucción tridimensional. Sin embargo, no hay estudios que comparen el mapeo tridimensional con la ablación convencional.
De otro lado, la ablación de pacientes con miocardiopatía hipertrófica y taquicardia ventricular monomórfica debe llevarse a cabo en centros con experiencia en mapeo tridimensional, acceso endoepicárdico percutáneo, disponibilidad inmediata de cirugía cardiovascular y experticia en ablación quirúrgica por radiofrecuencia y/o crioablación. Para pacientes sometidos a un segundo procedimiento con acceso epicárdico percutáneo, con toracotomía previa o evidencia de adherencias pericárdicas por métodos no invasivos, se debe considerar la posibilidad de ventana pericárdica subxifoidea o toracotomía para el acceso epicárdico cuando el acceso percutáneo fue fallido.

En la taquicardia ventricular en miocardiopatía hipertrófica se debe considerar el uso de imágenes diagnósticas preprocedimiento (TAC, RNM o estudios de medicina nuclear) para evaluar el sustrato arrítmico (grado de hipertrofia y fibrosis endo/epicárdica). Aunque la RNM cardiaca es el método de elección para valorar el sustrato arrítmico en miocardiopatía hipertrófica, la mayoría de los pacientes llevados a ablación de taquicardia ventricular con mapeo 3-D portan CDI y esto contraindica su uso. Sin embargo, hay alguna evidencia en centros especializados en los cuales la RNM cardiaca se ha empleado en forma rutinaria para valorar el sustrato arrítmico ventricular previo a ablación con mapeo 3-D. Estos centros emplean un protocolo específico para la obtención de RNM cardiaca y guías para la programación y seguimiento del CDI antes y después de la RNM. En el seguimiento a largo plazo no se han demostrado complicaciones relacionadas con disfunción o alteración del CDI ${ }^{15,16}$.

En la actualidad se realizan avances tecnológicos para el desarrollo de CDI compatibles con sistemas de RNM (denominados sistemas RNM condicionales). Hoy día existe un modelo de CDI comercialmente disponible, compatible con RNM extracardiaca y se espera que en el futuro próximo haya modelos de CDI compatibles con RNM cardiaca.

\section{Taquicardia ventricular catecolaminérgica}

La taquicardia ventricular polimórfica catecolaminérgica es un síndrome arrítmico hereditario poco frecuente, caracterizado por taquicardia ventricular bidireccional y polimórfica inducidas por estimulación adrenérgica. Se estima que la prevalencia de esta enfermedad en Europa es de alrededor de $1: 10.000^{17}$ y se desconocen cifras para Colombia y Latinoamérica en general. Su presentación clínica corresponde a la de taquicardia ventricular polimórfica, la cual es fácil de inducir durante pruebas de esfuerzo, infusión de isoproterenol o adrenalina, estrés emocional o ejercicio.

Esta enfermedad puede ser transmitida de manera autosómica dominante o recesiva. La mitad de los casos por transmisión autosómica dominante son causados por mutaciones en el gen que codifica el receptor de rianodina cardiaca (RyR2), responsable de la liberación de calcio por el retículo sarcoplasmático. La forma recesiva es causada por mutaciones en el gen que codifica la calsecuestrina (CASQ2), una proteína que retiene calcio en el retículo sarcoplasmático ${ }^{18,19}$.

La enfermedad se manifiesta en la infancia o adolescencia y puede ocasionar síncope y muerte súbita cardiaca a una edad temprana, en ausencia de enfermedad cardiaca estructural. El electrocardiograma (ECG) basal en reposo, incluyendo el intervalo QTc, es normal. La mortalidad por taquicardia 
ventricular polimórfica catecolaminérgica es extremadamente alta, llegando hasta el $31 \%$ a la edad de 30 años, si no es tratada. En una serie de pacientes con este diagnóstico, quienes no recibieron tratamiento alguno, se observaron eventos cardiacos mayores (muerte súbita cardiaca, síncope y/o taquicardia ventricular polimórfica) en el 33 y $58 \%$ de los pacientes a los 4 y 8 años de seguimiento respectivamente. Existe una correlación estrecha entre la edad de presentación de los síntomas y la severidad de la enfermedad, con peor pronóstico para aquellos con presentación temprana de arritmias o síncope ${ }^{20}$.

La prueba de esfuerzo desempeña un papel diagnóstico importante. Una vez la frecuencia cardiaca llega a 120-130 latidos por minuto (lpm), se comienzan a observar complejos ventriculares prematuros aislados, los cuales progresan a episodios de taquicardia ventricular no sostenida al aumentar la carga de ejercicio, y finalmente al desarrollo de taquicardia ventricular sostenida, la cual se caracteriza por una alternancia en el eje del QRS de 180 grados (taquicardia ventricu(ar bidireccional), aunque algunos pacientes pueden presentar taquicardia ventricular polimórfica o fibrilación ventricular en lugar de taquicardia ventricular bidireccional $^{21}$. El estudio electrofisiológico carece de utilidad diagnóstica o pronóstica y puede ser completamente normal, sin arritmias inducibles, en pacientes con taquicardia ventricular polimórfica catecolaminérgica.

Los pacientes que la padecen tienen alto riesgo de presentar arritmias ventriculares, cuyo desencadenante es el ejercicio, el cual ocasiona una descarga simpática que favorece el desarrollo de arritmias ventriculares polimórficas. Como no existe un sustrato arrítmico anatómico para la iniciación y perpetuación de dichas arritmias, el manejo se basa en la supresión farmacológica o quirúrgica del influjo simpático cardiaco. El primero se fundamenta en el uso de betabloqueadores, los cuales han demostrado un beneficio en la prevención de muerte súbita cardiaca y síncope de origen arrítmico ${ }^{22}$. Adicionalmente, en los últimos años se ha demostrado que la adición de otros antiarrítmicos como los bloqueadores de los canales del calcio ${ }^{23,24}$ y flecainida ${ }^{25,26}$ pueden disminuir el riesgo de arritmias en pacientes que no respondan al manejo convencional con betabloqueadores. Aquellos que sobreviven a un paro cardíaco de origen arrítmico o síncope arrítmico por taquicardia ventricular polimórfica catecolaminérgica son candidatos a $\mathrm{CDI}$ para prevención secundaria de muerte súbita ${ }^{27}$. Finalmente, la denervación simpática cardiaca izquierda ${ }^{28-30}$ desempeña un papel emergente en el manejo de esta patología.

En esta patología no se ha reportado la ablación, sin embargo existe un reporte de caso recientemente publicado ${ }^{31}$ de ablación de extrasístoles ventriculares bidireccionales desencadenando fibrilación ventricular en un paciente con diagnóstico de taquicardia ventricular polimórfica catecolaminérgica. Pese a ello, esta modalidad terapéutica no ha sido aún investigada de manera sistemática o reproducible.

\section{Síndrome de Brugada}

Fue descrito por primera vez en 1992 por los hermanos españoles Pedro y Josep Brugada, en asociación con un patrón electrocardiográfico de bloqueo de rama derecha y elevación del ST cóncavo en individuos jóvenes con corazón estructu- ralmente sano y fibrilación ventricular a partir de una serie de ocho pacientes ${ }^{32}$.

Se propusieron los criterios para el diagnóstico de síndrome de Brugada en 2002, los cuales se actualizaron en 2005 en el segundo documento de consenso. El diagnóstico requiere la presencia de elevación del punto $\mathrm{J}$ de $2 \mathrm{~mm}$ o más, con elevación del segmento $\mathrm{ST}$, e inversión de la onda $\mathrm{T}$, conocido como el patrón de tipo 1 electrocardiográfico, en dos o más de las derivaciones precordiales derechas V1-323,33.

Estos hallazgos pueden ser incidentales o inducidos por un bloqueador de los canales de sodio en combinación con al menos uno de los siguientes criterios: fibrilación ventricular/ taquicardia ventricular polimórfica documentada, historia familiar de muerte súbita cardiaca en miembros menores de 45 años de edad, un electrocardiograma tipo 1 en miembros de la familia, inducibilidad en la estimulación eléctrica programada, síncope o respiración agónica nocturna ${ }^{32,33}$. El típico patrón de Brugada en el electrocardiograma cuando no es manifiesto, puede ser revelado por antiarrítmicos de la clase IC que bloquean el componente rápido de las corrientes de los canales de sodio. El síndrome también puede ser desenmascarado o precipitado por un estado febril, agentes vagotónicos, agonistas adrenérgicos, bloqueadores betaadrenérgicos, antidepresivos tricíclicos o tetracíclicos, una combinación de glucosa e insulina e hipopotasemia, así como por alcohol y toxicidad por cocaína ${ }^{32}$. Una preocupación potencial es el papel de la inducibilidad en el estudio electrofisiológico como una herramienta de diagnóstico, dado el riesgo de falsos positivos en individuos normales ${ }^{33,34}$.

El mecanismo de aparición del síndrome de Brugada tradicionalmente se ha vinculado a alteraciones de la repolarización (fase 2 de reentrada), pero en los últimos años se han demostrado otros tipos de anormalidades en la despolarización ${ }^{35}$.

En los últimos 15 años, los estudios genéticos moleculares permitieron describir trastornos hereditarios arritmogénicos y mutaciones, principalmente en el gen SCN5A que codifica para la a-subunidad del canal de sodio, orientando hacia la etiología en el síndrome ${ }^{36-38}$.

De igual forma, este ha sido subdiagnosticado dada su presentación clínica variable. En los hombres del sudeste asiático, ya se había descrito la muerte súbita durante el sueño, conocida como el síndrome de muerte súbita inesperada, y en una serie, el $60 \%$ de los casos pudo atribuirse a esta patología 34,37 .

En la literatura médica se han publicado desde hace veinte años numerosos casos clínicos y series de casos que describen la variabilidad de su presentación, indican la importancia del implante de un CDI para prevención primaria de muerte súbita cardiaca, el uso de antiarrítmicos y la ablación por radiofrecuencia en casos refractarios a la terapia anterior ${ }^{39-41}$.

Se ha encontrado que el sitio del sustrato arrítmico en el síndrome de Brugada es el tracto de salida del ventrículo derecho, sea sobre el epicardio o endocardio, ya que se identificaron electrogramas anormales en esta ubicación, lo cual serviría como objetivo para la ablación con catéter. El mecanismo electrofisiológico subyacente es por retraso en la despolarización sobre la cara anterior del epicardio en dicha localización. Es importante reconocer que la ablación epicárdica para modificar el sustrato electrofisiológico puede tener sus limitaciones ya que la prolongación de electrogramas es dinámica ${ }^{42,43}$. 
En los pacientes sintomáticos con síndrome de Brugada, el CDI es la única opción para prevenir la muerte súbita. En los pacientes asintomáticos con un patrón de Brugada electrocardiográfico, la estratificación del riesgo es esencial para evaluar quiénes realmente necesitan tratamiento definitivo.

La ablación se ha usado en aquellos casos en los que la terapia médica no ha logrado evitar las tormentas arrítmicas recurrentes. Se han publicado varios informes de casos exitosos en la supresión de las tormentas de fibrilación ventricular mediante ablación endocárdica cuando las extrasístoles ventriculares son disparadores a nivel de Purkinje o el tracto de salida del ventrículo derecho ${ }^{34,37,42}$.

Aun así, debe tenerse presente que se han descrito casos de muerte súbita cardíaca diez semanas después de la ablación, lo cual sugiere que la normalización a corto plazo de repolarización ventricular puede ser de naturaleza transitoria y no es un indicador fiable de protección contra la muerte súbita cardiaca y de ahí la necesidad del seguimiento estricto de estos pacientes ${ }^{44}$.

Nademanee et al. ${ }^{39}$ y Morita et al. ${ }^{44}$ estudiaron la ablación por radiofrecuencia epicárdica para el tratamiento de arritmias ventriculares dada la heterogeneidad en la morfología y la duración de potenciales de acción a este nivel, en contraste con electrogramas normales en el endocardio. Encontraron que la ablación de la zona epicárdica de los potenciales anormales resultó en la normalización del patrón de Brugada en siete de nueve pacientes. Sin embargo, pese al cambio a largo plazo en el sustrato arrítmico y la desaparición del patrón de Brugada electrocardiográfico, son aparentemente ineficaces en la eliminación de extrasístoles ventriculares a corto plazo ${ }^{45}$.

La ablación en el tracto de salida del ventrículo derecho y la red de Purkinje han demostrado eliminar el sustrato de la taquicardia ventricular/fibrilación (VT/VF) en síndrome de Brugada para abortar tormentas eléctricas ${ }^{46}$. Las zonas de activación tardía, identificadas por cartografía pueden servir como sustrato potencial de fibrilación ventricular en dicho síndrome y son los objetivos de la ablación. El procedimiento es seguro y puede prevenir la aparición de la fibrilación ventricular. Cabe resaltar que no hay información del tratamiento en niños ${ }^{47}$. Es necesario utilizar técnicas de mapeo tridimensional para realizar una valoración adecuada del sustrato arrítmico y definir los sitios de ablación.

En la actualidad no se cuenta con datos con relación a la ablación profiláctica ni respecto a si esta es capaz de sustituir a la terapia con CDI.

\section{Recomendaciones}

\section{Miocardiopatía hipertrófica}

\section{Clase I (nivel de evidencia C)}

- La ablación con mapeo 3-D está indicada como terapia adicional en pacientes con miocardiopatía hipertrófica quienes presenten tormenta arrítmica, definida como taquicardia ventricular recurrente tratada con descargas apropiadas del CDI, ATP (terapia antitaquicardia) o taquicardia ventricular recurrente lenta no tratada (debajo de la zona de detección) a pesar de manejo adecuado de falla cardiaca, tratamiento antiarrítmico, cambios en la progra- mación del dispositivo o que requieran la suspensión de betabloqueadores $u$ otros medicamentos antiarrítmicos por desarrollar efectos adversos. La ablación debe llevarse a cabo con técnicas de mapeo tridimensional y acceso endoepicárdico percutáneo para evaluar el sustrato tanto epicárdico como endocárdico y garantizar el mayor grado de éxito y control de la taquicardia ventricular ( $\approx 70 \%)$.

\section{Clase lla (nivel de evidencia C)}

- La ablación con mapeo 3-D está indicada como terapia adicional en pacientes con miocardiopatía hipertrófica quienes presenten tormenta arrítmica (definida como taquicardia ventricular recurrente tratada con descargas apropiadas del CDI, ATP o taquicardia ventricular recurrente lenta no tratada debajo de la zona de detección) a pesar de manejo adecuado de falla cardiaca, tratamiento antiarrítmico, cambios en la programación del dispositivo o que requieran la suspensión de betabloqueadores u otros medicamentos antiarrítmicos por desarrollar efectos adversos. La ablación debe llevarse a cabo con técnicas de mapeo tridimensional y se acepta el mapeo y ablación endocárdico exclusivo para pacientes críticos que no hayan respondido a las medidas anteriores y no se encuentren en un centro con experiencia en mapeo y ablación epicárdica y/o sin experiencia en ablación quirúrgica por radiofrecuencia y/o crioablación, aceptando una menor posibilidad de control agudo de taquicardia ventricular y un mayor porcentaje de recurrencias a largo plazo.

- Se acepta la ablación endocárdica guiada por mapeo 3-D (para centros sin experiencia en ablación epicárdica) o endo/epicárdica guiada por mapeo 3-D (para centros con experiencia en ablación epicárdica) para pacientes con diagnóstico de miocardiopatía hipertrófica quienes sufran tormenta ventricular a pesar del manejo adecuado de la falla cardiaca y tratamiento antiarrítmico, con el fin de controlar la carga arrítmica como puente al implante de un CDI para prevención secundaria de muerte súbita.

\section{Clase IIb (nivel de evidencia $C$ )}

- Se puede considerar la ablación endocárdica guiada por mapeo 3-D (para centros sin experiencia en ablación epicárdica) o endo/epicárdica guiada por mapeo 3-D (para centros con experiencia en ablación epicárdica) para pacientes con diagnóstico de miocardiopatía hipertrófica portadores de CDI, quienes sufran tormenta ventricular que no responda al manejo antiarrítmico, a pesar de no recibir manejo óptimo para falla cardiaca (incluye pacientes con presentación de novo de falla cardiaca y aquellos con pobre adherencia al manejo ambulatorio de falla cardiaca).

\section{Clase III (nivel de evidencia C)}

- La ablación guiada por mapeo 3-D NO está indicada en pacientes con miocardiopatía hipertrófica y taquicardia ventricular recurrente, quienes no estén recibiendo tratamiento farmacológico óptimo con betabloqueadores y antiarrítmicos (salvo en aquellos casos que tengan una contraindicación absoluta para recibir los mismos).

- La ablación con acceso endocárdico exclusivo guiada por mapeo 3-D NO está indicada en pacientes con miocardio- 
patía hipertrófica y taquicardia ventricular recurrente luego de un primer procedimiento de ablación fallido, posterior a un procedimiento de ablación exitosa con recurrencia o cuando la sospecha clínica de taquicardia ventricular con foco epicárdico sea alta con base en el electrocardiograma de superficie y/o en imágenes diagnósticas (ecocardiografía, RNM, TAC o estudios de medicina nuclear).

\section{Taquicardia ventricular catecolaminérgica}

\section{Clase I, Ila y IIb (nivel de evidencia C)}

- No hay.

\section{Clase III (nivel de evidencia C)}

- Las técnicas de mapeo tridimensional y la ablación por radiofrecuencia o crioablación no están indicadas para el manejo de arritmias ventriculares relacionadas con la taquicardia ventricular catecolaminérgica.

\section{Síndrome de Brugada}

\section{Clase Ila (nivel de evidencia C)}

- La ablación guiada por mapeo 3-D está indicada en pacientes con diagnóstico de Síndrome de Brugada, que tengan implantado un CDI y que presenten tormenta arrítmica que no mejore con tratamiento antiarrítmico o uso de isoproterenol.

- La realización de ablación guiada por mapeo 3-D en síndrome de Brugada debe realizarse en centros con experiencia en mapeo epicárdico.

\section{Bibliografía}

1. Maron BJ, Maron MS. Hypertrophic cardiomyopathy. Lancet. 2013;381(9862):242-55.

2. Alcalai R, Seidman JG, Seidman CE. Genetic basis of hypertrophic cardiomyopathy: from bench to the clinics. J Cardiovasc Electrophysiol. 2008;19:104-10.

3. Maron BJ, McKenna WJ, Danielson GK, Kappenberger LJ, Kuhn HJ, Seidman CE, et al.; Task Force on Clinical Expert Consensus Documents. American College of Cardiology; Committee for Practice Guidelines. European Society of Cardiology. American College of Cardiology/European Society of Cardiology clinical expert consensus document on hypertrophic cardiomyopathy. A report of the American College of Cardiology Foundation Task Force on Clinical Expert Consensus Documents and the European Society of Cardiology Committee for Practice Guidelines. J Am Coll Cardiol. 2003;42:1687-713.

4. Seidman CE, Seidman JG. Identifying sarcomere gene mutations in hypertrophic cardiomyopathy: a personal history. Circ Res. 2011;108:743-50.

5. Ackerman MJ, VanDriest SL, Ommen SR, et al. Prevalence and age-dependence of malignant mutations in the beta-myosin heavy chain and troponin T genes in hypertrophic cardiomyopathy: a comprehensive outpatient perspective. J Am Coll Cardiol. 2002;39:2042-48.

6. Maron BJ, Spirito P, Shen WK, et al. Implantable cardioverterdefibrillators and prevention of sudden cardiac death in hypertrophic cardiomyopathy. JAMA. 2007;298:405-12.

7. Maron BJ, Doerer JJ, Haas TS, et al. Sudden deaths in young competitive athletes: analysis of 1866 deaths in the United States, 1980-2006. Circulation. 2009;119:1085-92.
8. Maron BJ, Maron MS, Lesser JR, et al. Sudden cardiac arrest in hypertrophic cardiomyopathy in the absence of conventional criteria for high risk status. Am J Cardiol. 2008;101:54447.

9. Maron BJ, McKenna WJ, Danielson GK, Kappenberger LJ, Kuhn HJ, Seidman CE, et al.; Task Force on Clinical Expert Consensus Documents. American College of Cardiology; Committee for Practice Guidelines. European Society of Cardiology. American College of Cardiology/European Society of Cardiology clinical expert consensus document on hypertrophic cardiomyopathy. A report of the American College of Cardiology Foundation Task Force on Clinical Expert Consensus Documents and the European Society of Cardiology Committee for Practice Guidelines. J Am Coll Cardiol. 2003;42:1687-713.

10. Gersh BJ, Maron BJ, Bonow RO, et al. 2011 ACCF/AHA guidelines for the diagnosis and treatment of hypertrophic cardiomyopathy: a report of the American College of Cardiology Foundation/American Heart Association Task Force on practice guidelines. J Am Coll Cardiol. 2011;58:212-60.

11. Rickers $C$, Wilke NM, Jerosch-Herold $M$, et al. Utility of cardiac magnetic resonance imaging in the diagnosis of hypertrophic cardiomyopathy. Circulation. 2005;112:855-61.

12. Dukkipati SR, d'Avila A, Soejima K, et al. Long-term outcomes of combined epicardial and endocardial ablation of monomorphic ventricular tachycardia related to hypertrophic cardiomyopathy. Circ Arrhythm Electrophysiol. 2011;4:185-94.

13. Della Bella P, Brugada J, Zeppenfeld K, et al. Epicardial ablation for ventricular tachycardia: a European multicenter study. Circ Arrhythm Electrophysiol. 2011;4:653-9.

14. Cano $O$, Hutchinson $M$, Lin $D$, et al. Electroanatomic substrate and ablation outcome for suspected epicardial ventricular tachycardia in left ventricular nonischemic cardiomyopathy. J Am Coll Cardiol. 2009;54:799-808.

15. Dickfeld T, Tian J, Ahmad G, et al. MRI-Guided ventricular tachycardia ablation: integration of late gadolinium-enhanced 3D scar in patients with implantable cardioverter-defibrillators. Circ Arrhythm Electrophysiol. 2011; 4:172-84.

16. Nazarian S, Hansford R, Roguin A, et al. A prospective evaluation of a protocol for magnetic resonance imaging of patients with implanted cardiac devices. Ann Intern Med. 2011;155:415-24.

17. Pflaumer A, Davis AM. Guidelines for the diagnosis and management of Catecholaminergic Polymorphic Ventricular Tachycardia. Heart Lung Circ. 2012;21:96-100.

18. Priori SG, Napolitano C, Memmi M, et al. Clinical and molecular characterization of patients with catecholaminergic polymorphic ventricular tachycardia. Circulation. 2002;106:69-74.

19. Liu N, Rizzi N, Boveri L, Priori SG. Ryanodine receptor and calsequestrin in arrhythmogenesis: whatwe have learnt from genetic diseases and transgenic mice. J Mol Cell Cardiol. 2009;46:149-59.

20. Leenhardt A, Denjoy I, Guicheney P. Catecholaminergic polymorphic ventricular tachycardia. Circ Arrhythm Electrophysiol. 2012;5:1044-52.

21. Fisher JD, Krikler D, Hallidie-Smith KA. Familial polymorphic ventricular arrhythmias: a quarter century of successful medical treatment based on serial exercise-pharmacologic testing. J Am Coll Cardiol. 1999;34:2015-22.

22. Van der Werf C, Zwinderman AH, Wilde AA. Therapeutic approach for patients with catecholaminergic polymorphic ventricular tachycardia: state of the art and future developments. Europace. 2012;14:175-83.

23. Rosso R, Kalman JM, Rogowski O, et al. Calcium channel blockers and beta-blockers versus beta-blockers alone for preventing exercise-induced arrhythmias in catecholaminergic polymorphic ventricular tachycardia. Heart Rhythm. 2007;4: 1149-54.

24. Swan H, Laitinen P, Kontula K, et al. Calcium channel antagonism reduces exercise-induced ventricular arrhythmias in cate- 
cholaminergic polymorphic ventricular tachycardia patients with RyR2 mutations. J Cardiovasc Electrophysiol. 2005; $16: 162-6$.

25. Watanabe H, Chopra N, Laver D, Hwang HS, Davies SS, Roach $\mathrm{DE}$, et al. Flecainide prevents catecholaminergic polymorphic ventricular tachycardia in mice and humans. Nat Med. 2009;15:380-3.

26. Van der Werf C, Kannankeril PJ, Sacher F, Krahn AD, Viskin S, Leenhardt A, et al. Flecainide therapy reduces exercise-induced ventricular arrhythmias in patients with catecholaminergic polymorphic ventricular tachycardia. J Am Coll Cardiol. 2011; 57:2244-54.

27. Zipes DP, Camm AJ, Borggrefe M, et al. ACC/AHA/ESC 2006 Guidelines for Management of Patients With Ventricular Arrhythmias and the Prevention of Sudden Cardiac Death: a report of the American College of Cardiology/American Heart Association Task Force and the European Society of Cardiology Committee for Practice Guidelines (writing committee to develop Guidelines for Management of Patients With Ventricular Arrhythmias and the Prevention of Sudden Cardiac Death): developed in collaboration with the European Heart Rhythm Association and the Heart Rhythm Society. Circulation. 2006; 114:e385-484.

28. Collura CA, Johnson JN, Moir C, Ackerman MJ. Left cardiac sympathetic denervation for the treatment of long QT syndrome and catecholaminergic polymorphic ventricular tachycardia using video-assisted thoracic surgery. Heart Rhythm. 2009;6:752-9.

29. Atallah J, Fynn-Thompson F, et al. Video-assisted thoracoscopic cardiac denervation: a potential novel therapeutic option for children with intractable ventricular arrhythmias. Ann Thorac Surg. 2008;86:1620-5.

30. Wilde AA, Bhuiyan ZA, Crotti L, Facchini M, De Ferrari GM, Paul $\mathrm{T}$, et al. Left cardiac sympathetic denervation for catecholaminergic polymorphic ventricular tachycardia. New Engl J Med. 2008;358:2024-9.

31. Kaneshiro T, Naruse Y, Nogami A, et al. Successful catheter ablation of bidirectional ventricular premature contractions triggering ventricular fibrillation in catecholaminergic polymorphic ventricular tachycardia with RyR2 mutation. Circ Arrhythm Electrophysiol. 2012;5:e14-7.

32. Brugada $P$, Benito $B$, Brugada R, Brugada J. Brugada syndrome: update 2009. Hellenic J Cardiol. 2009;50:352-72.

33. Boussy T, Sarkozy A, Brugada P. The Brugada syndrome: update 2006. Arch Mal Coeur Vaiss. 2007;100:865-71.

34. Li A, Behr ER. Brugada syndrome: an update. Future Cardiol. 2013;9:253-71.
35. Sacher F, Jesel L, Jais $P$, Haissaguerre $M$. Insight into the mechanism of Brugada syndrome: epicardial substrate and modification during ajmaline testing. Heart Rhythm 2014;11:732-4.

36. Hsiao PY, Tien HC, Lo CP, et al. Gene mutations in cardiac arrhythmias: a review of recent evidence in ion channelopathies. Appl Clin Genet. 2013;6:1-13.

37. Veerakul G, Nademanee K. Brugada syndrome: two decades of progress. Circ J. 2012;76:2713-22.

38. Lippi G, Montagnana M, Meschi T, Comelli I, Cervellin G. Genetic and clinical aspects of Brugada syndrome: an update. Adv Clin Chem. 2012;56:197-208.

39. Nademanee K, Veerakul G, Chandanamattha P, et al. Prevention of ventricular fibrillation episodes in Brugada syndrome by catheter ablation over the anterior right ventricular outflow tract epicardium. Circulation. 2011;123:1270-9.

40. Nakagawa E, Takagi M, Tatsumi H, Yoshiyama M. Successful radiofrequency catheter ablation for electrical storm of ventricular fibrillation in a patient with Brugada syndrome. Circ J. 2008;72:1025-9.

41. Szeplaki G, Ozcan EE, Osztheimer I, Tahin T, Merkely B, Geller $\mathrm{L}$. Ablation of the epicardial substrate in the right ventricular outflow tract in a patient with Brugada syndrome refusing implantable cardioverter defibrillator therapy. Can J Cardiol. 2014;30:1249.e9-1249.e11.

42. Haissaguerre $M$, Extramiana $F$, Hocini $M$, Cauchemez $B$, Jais $P$, Cabrera JA, et al. Mapping and ablation of ventricular fibrillation associated with long-QT and Brugada syndromes. Circulation. 2003;108:925-8.

43. Brugada J, Berruezo A, Cuesta A, Osca J, Chueca E, Fosch X, et al. Nonsurgical transthoracic epicardial radiofrequency ablation: an alternative in incessant ventricular tachycardia. J Am Coll Cardiol. 2003;41:2036-43.

44. Morita H, Zipes DP, Morita ST, Lopshire JC, Wu J. Epicardial ablation eliminates ventricular arrhythmias in an experimental model of Brugada syndrome. Heart Rhythm. 2009;6:665-71.

45. Shan Q, Yang B, Chen M, Zou J, Chen C, Xu D, et al. Short-term normalization of ventricular repolarization by transcatheter ablation in a patient with suspected Brugada syndrome. J Interv Card Electrophysiol. 2008;21:53-7.

46. Sunsaneewitayakul B, Yao Y, Thamaree S, Zhang S. Endocardial mapping and catheter ablation for ventricular fibrillation prevention in Brugada syndrome. J Cardiovascular Electrophysiol. 2012;23 Suppl 1:S10-6.

47. Conte G, Chierchia GB, Levinstein M, Brugada P. Ice vs. fire: cryoballoon ablation for the prevention of inappropriate implantable cardioverter-defibrillator shocks in a 14-year-old girl with Brugada syndrome. Europace. 2013;15:1804. 